\title{
Exploring the Structure of Social Media Network with the Concepts of Graph Theory
}

\author{
Kamal Jyoti Barman, Kuntala Patra
}

\begin{abstract}
Millions of people use online social networking sites such as Facebook, Twitter, WhatsApp, Instagram etc. Nowadays, social media is very popular among all of us especially young generation and has become a vital part of our life. Just like a graph is made up of vertices and edges, a social media network is made up of persons or communities where each person or community represents the vertex of the social graph and the adjacency of the vertices (edges) is determined via friendship, common interest, common liking etc. In this paper we try to show the use of some graphical parameter in social graph so that the many properties of social media network can be reflected through graph. We have also shown how domination number plays an important role in social graph.
\end{abstract}

Keywords : Social media, Social media network, Social graph , Facebook. 2010 MSC No : 05C82 , 68R10, 94C15.

\section{INTRODUCTION}

Social media plays a vital role in communicating people all over the world and is extremely popular among young generation. It enables the user to share information and upload/download images and videos on the internet. A social network is a social structure composed of a set of social units ( like individuals or organizations ) and social interactions among them [6]. Just like a graph is made up of vertices and edges, a social media network is made up of persons or communities where each person or community represents a vertex and the adjacency of the vertices ( edges ) is determined via friendship, common interest, common likes etc. A social graph is a graph that represents interconnection among people, groups and organizations in a social network [6]. In short , social graph is a graphical representation of social network with the help of graph theory. Social media is considered as an effective marketing/communication tool for corporation , entrepreneurs, political parties , organizations etc. Since the overall graphical structure of social media is very complex with millions of units and interconnections among them, so for our convenience we study only a part of a graph ( sub graph ) instead of the whole graph.

Revised Manuscript Received on April 13, 2020.

* Correspondence Author

Kamal Jyoti Barman*, PhD Research Scholar, Department of Mathematics, Gauhati University, Guwahati-781014.

Kuntala Patra, Professor , Department of Mathematics , Gauhati University, Guwahati-781014.

(C) The Authors. Published by Blue Eyes Intelligence Engineering and Sciences Publication (BEIESP). This is an open access article under the CC BY-NC-ND license (http://creativecommons.org/licenses/by-nc-nd/4.0/)

\section{II.SOME PRELIMINARY DEFINITIONS}

1. A graph $G$ consists of a finite non-empty set $V(G)$ of points/vertices together with a prescribed set $E(G)$ of unordered pairs of distinct points of $V(G)$, where each pair of points denotes an edge.

2. A subgraph of $G$ is a graph having all of its points and lines in $G$.

3. A graph is connected if every pair of points are joined by a path. Otherwise if there exist a pair of points which are not joined by a path then the graph is said to be disconnected.

4. A graph $G$ having no lines is known as totally disconnected graph.

5. A graph $G$ with $n$ vertices is said to be complete graph if every pair of vertices are joined by an edge. It is denoted by $K_{n}$.

6. A star graph is a graph with $n$ vertices such that exactly one vertex has degree $n-1$ and the remaining $n-1$ vertices have degree 1.

7. A dominating set for a graph $G=(V, E)$ is a subset $D$ of $V$ such that every vertex not in $D$ is adjacent to atleast one member of $D$. The domination number, $\gamma(G)$ is the number of vertices in a smallest dominating set for $G$.

\section{III.MEASURE OF INFLUENCE IN A SOCIAL GRAPH}

The influence of a person in social media is directly proportional to the connectivity of vertex to that person in a social graph. So, for a graph $G$, where edge relationship is determined by friendship, the person having lots of friends tend to have more influence.

Let for a person $p$, the measure of his/her influence is denoted by $I(p)$ and is defined as $I(p)=\operatorname{deg}(p)$ in $G$

$$
=\text { Number of friends of person } p \text {. }
$$

Which can be easily found by simply looking at the vertex corresponding to the person $p$ in a graph $G$.

Published By:

Blue Eyes Intelligence Engineering

\& Sciences Publication

(C) Convriaht: All riahts reserved 


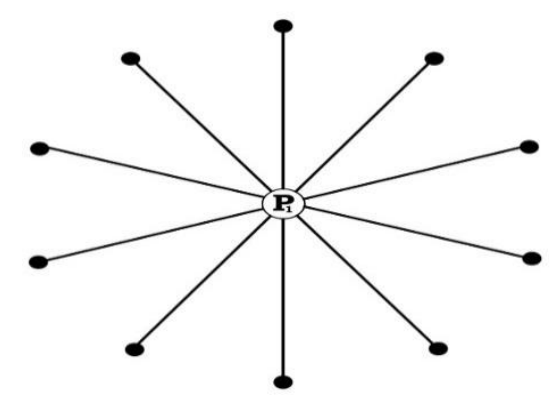

Fig : 1

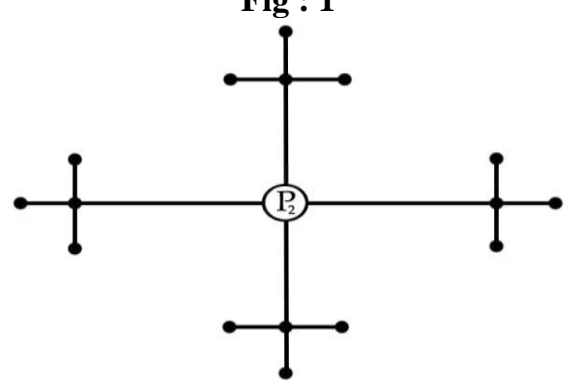

Fig : 2

The person $p_{1}$, has higher measure of influence because person $p_{1}$ is directly connected with 10 people while person $p_{2}$ is directly connected to 4 people only but person $p_{2}$ has the potential to influence up to 16 people.

This happens in real life too, e.g. the CEO of a company has direct relationships with Board of directors, Vice-president and some employees, yet he/she is more influential than the others.

Hence, we can modify the definition for measure of influence of a person $p, I(p)$ as , $I(p)=$ Sum of the degre e ${ }^{I}$ s of all persons who are directly connected with person isk. $^{2}$.

Degree of the person $p$

So, from above figures, measure of influence of person $p_{1}$,

$$
I\left(p_{1}\right)=\frac{1+\cdots+1(10 \text { times })}{10}
$$$$
=1
$$

and measure of influence of person $p_{2}$, $I\left(p_{2}\right)=\frac{4+4+4+4}{4}$

$$
=4
$$

Implying, person $p_{2}$ is more influential than the person $p_{1}$.

\section{IV.STRONG , AVERAGE AND WEAK CONNECTION IN A SOCIAL GRAPH}

Any two persons $p_{1}$ and $p_{2}$ in a social media knows each other because either they are friends or they are the member of same communities. These communities may be a group of businessman, group of scientists etc.

Suppose $p_{1}$ and $p_{2}$ are two persons such that both of them are friends as well as member of same community $C_{1}$. Graphically we represent it as follows :

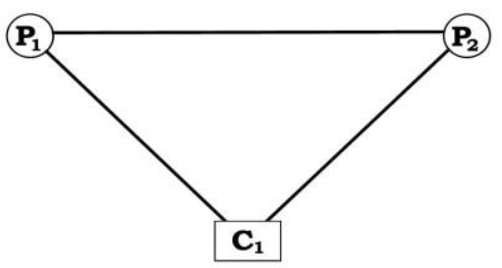

Fig : 3

Then we say that connection between person $p_{1}$ and person $p_{2}$ is strong, as they are connected via two different paths, one is from friendship and other one is from same community. If person $p_{1}$ and person $p_{2}$ are friends and connected through that path only then we say that the connection between them is average.

Else, if they are not friends but connected via two different path viz : through same community and common friend, then also we say that the connection between them is average.

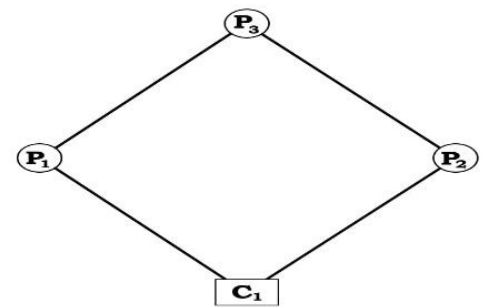

Fig : 4

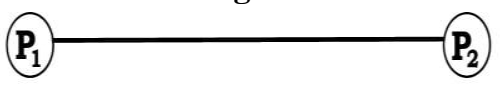

Fig : 5

And if person $p_{1}$ and person $p_{2}$ are connected through one path only, which is either through same community or common friend, then we say that the connection between them

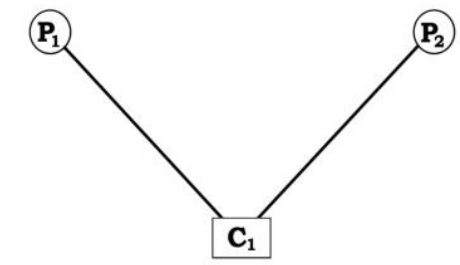

Fig : 6

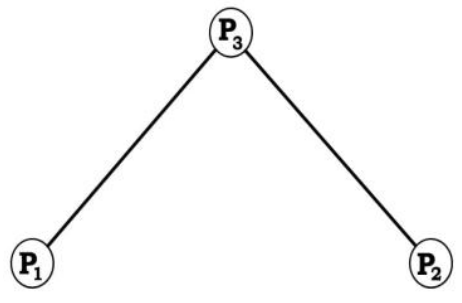

Fig : 7

\section{V.SINGLE - CONNECTED AND MULTI - CONNECTED SOCIAL GRAPH}

In a social graph consisting of different groups of people, sometimes it may happen that a single person from either of the groups can not establish a connection between two different groups of people. But he/she might have a friend who is connected with a single person of another group.

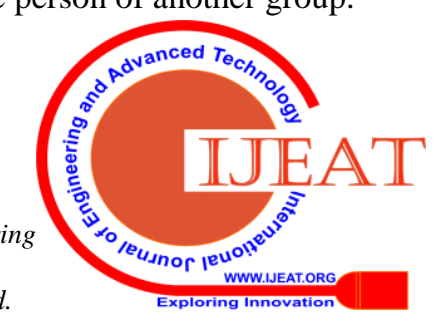


In such a case, we say that groups $G_{1}$ and $G_{2}$ in a social graph are single-connected and the path through which they are connected is known as Duet-bridge.

For example :

Suppose $G_{1}$ is a group of students of a college $C_{1 \text { and }} G_{2}$ is a group of students of college $C_{2}$ such that no two students of college $C_{1}$ and college $C_{2}$ are friends with each other.

Let $p_{1}$ be a student of college $C_{1}$ and $p_{2}$ be a friend of $p_{1}$ such that $p_{2}$ has another friend $p_{3}$ from college $C_{2}$. In this case the students of two colleges are connected in social media with the help of person $p_{2}$.

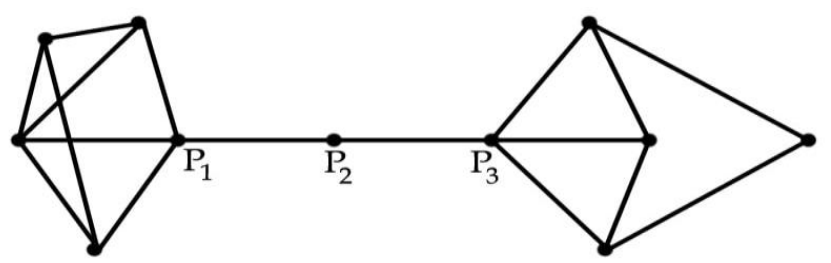

Fig : 8

Here $p_{1}-p_{2}-p_{3}$ is Duet-bridge.

If there are more than one Duet-bridge present between two groups $G_{1}$ and $G_{2}$ in a social graph $G$ then we say that the groups $G_{1}$ and $G_{2}$ are multi-connected in a social graph $G$.

If the two groups have only one Duet-bridge in a social graph, then that Duet-bridge is the only way to get the information from each other. Sometimes it may happened that the social unit of that Duet-bridge do not share the complete or exact informations, so it will be better if there is another Duet-bridge present between the two groups.

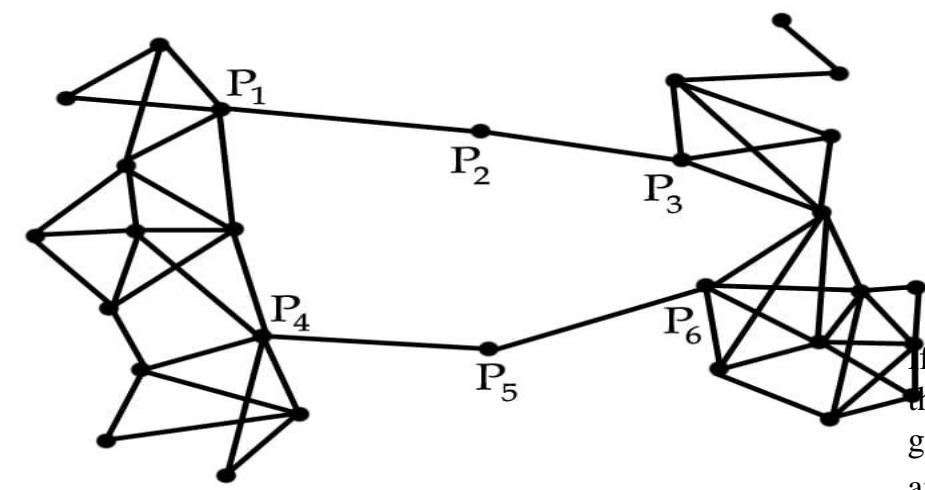

$\mathbf{G}_{1}$

$G_{2}$

Fig : 9

Nowadays nobody can believe in single person or single social unit and so it is better to collect information about other group if the groups are multi-connected in a social graph. Moreover, if there are more than one Duet-bridge present between any two groups in a social graph then people may be confused regarding which one is better of getting new information. In that case, if a Duet-bridge is connected to a person with high measure of influence then that Duet-bridge will transfer more and accurate information to another group. Otherwise if a Duet-bridge is connected to a person with low measure of influence then that Duet-bridge will transfer little/less information to another group. For example : In above figure, group $G_{1}$ wants to know some new information from group $G_{2}$. For that we have two Duet-bridge viz : $p_{1}-p_{2}-p_{3}$ and $p_{4}-p_{5}-p_{6}$. Now we find the measure of influence of person $p_{3}$ and $p_{6}$ of group $G_{2}$.

$$
\begin{array}{r}
I\left(p_{3}\right)=\frac{2+4+3+7}{4}=4 \\
I\left(p_{6}\right)=\frac{2+6+4+6+7}{5}=5
\end{array}
$$

Since measure of influence of person $p_{6}$ is more as compared to the measure of influence of person $p_{3}$, so Duet-bridge $p_{4}-p_{5}-p_{6} \quad$ is preferred over Duet-bridge $p_{1}-p_{2}-p_{3}$ for collection of information from group $G_{2}$.

\section{VI.EDGE-CUT IN A SOCIAL GRAPH}

The definition of Bridge in graph theory [4] leads us to define Edge-cut in a social graph.

For a social graph, it will be convenient if we study the properties of sub graph ( part of a graph ) instead of whole graph to get the information more accurately and for that reason we introduce the concept of edge-cut in the graph of social network.

Edge-cut means removing such an edge from a graph of social network so that the graph becomes disconnected. Removing an edge from a social graph means that the connection between two vertices fails, which can be related to the fact that an individual has been removed from the friend list of another individual.

For example :

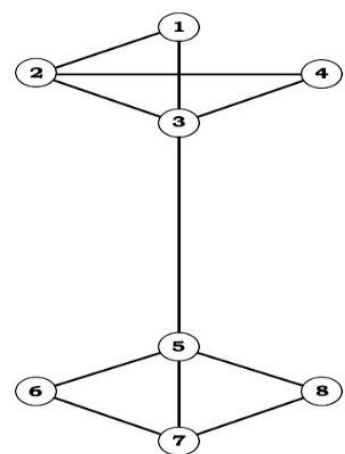

Fig : 10

an edge has been removed between person 3 and person 5 hen the group will be divided into two components or sub graphs. This edge is called the edge-cut of the social graph and it can be viewed as, if in a social graph $G$, either person 3 unfriend/remove person 5 or person 5 unfriend/remove person 3 from their friend list then the graph becomes disconnected. And as a result of this, the individual will no longer be able to view posts from friends of friend that means person 5 will not be able to see posts from person 3 as well as his/her friends person 1, person 2 and person 4 and vice-versa.

\section{VII.TRIANGULAR EDGE IN SOCIAL GRAPH}

The definition of Triadic closure given by [9] motivates us to define Triangular edge in a social graph. If an edge between the vertices $B$ and $C$ froms a triangle $A B C$ in a social graph $G$ then the edge $B C$ is known as Triangular edge.

Published By:

Blue Eyes Intelligence Engineering \& Sciences Publication

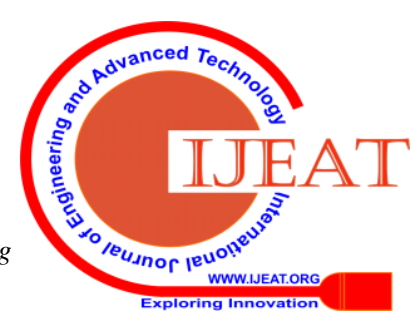


Consequence in a social media :

If two persons have a common friend in social media then there is an increased chance that they will become friends with each other in future.

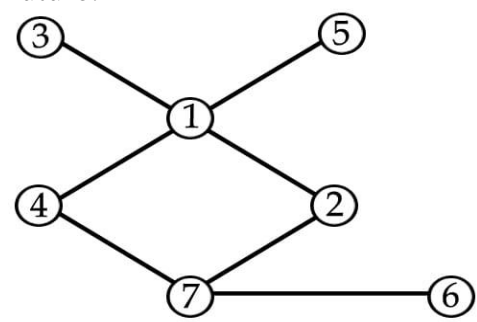

Fig : 11

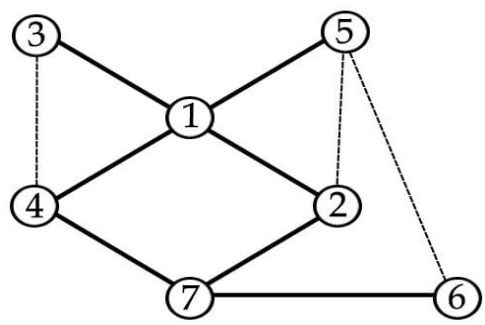

Fig : 12

Person 3 and person 4 have person 1 as a common friend and as a consequence of triangular edge they become friends. But all the new friendship may not be due to triangular edge e.g : person 5 and person 6 .

\section{VIII.FACEBOOK}

Suppose each person of university/college/office represents vertices of a graph $G$ ( assuming that all of them have Facebook accounts ) and any two distinct vertices $x$ and $y$ of $G$ are adjacent whenever person $x$ and person $y$ are friends. Then by looking at the graph $G$, following conclusion can be drawn :

$$
\begin{aligned}
& \text { 1. Let } x, y \in V(G) \text { then } \\
& \begin{array}{l}
d(x, y)=1 \Rightarrow \text { person } x \text { and person } y \text { are } \\
\text { friend. } \\
d(x, y)=2 \Rightarrow \text { person } x \text { and person } y \text { are not }
\end{array}
\end{aligned}
$$
friend but they have a mutual/common friend.

$d(x, y)>2 \Rightarrow$ person $x$ and person $y$ are neither a friend nor they have a mutual friend.

2. Let $x \in V(G)$ then

$\operatorname{deg}(x)=0 \Rightarrow$ person $x$ have no friends.

$\operatorname{deg}(x)=n=>$ person $x$ have $n$ friends.

3. Let $x_{1}, x_{2}, \ldots \ldots, x_{n} \in V(G)$

If $x_{1}, x_{2}, \ldots \ldots, x_{n}$ forms a complete graph $K_{n}$ in $G$ then person $x_{1}$, person $x_{2}, \ldots \ldots$, person $x_{n}$ are all friends with each other.

4. If girth of $G, \operatorname{gr}(G) \neq 3$ then no two friends have a common friend.

5. If diameter of $G, \operatorname{diam}(G)=1$ then all the members of university/college/office are friends with each other.

\section{HOW TO TAG MINIMUM NUMBER OF PERSON SO THAT THE POST IS AVAILABLE TO ALL}

Suppose someone from outside the university/college/office wants to share some information to all members by tagging the minimum number of person.

In that case all we need to do is to find the domination number of the graph $G$ corresponding to the university/college/office. If the domination number is $n$ that is dominating set contains $n$ - vertices then it is enough to tag those $n$ persons corresponding to the $n$ vertices present in the dominating set in a post so that the post is available to all of them. For example :

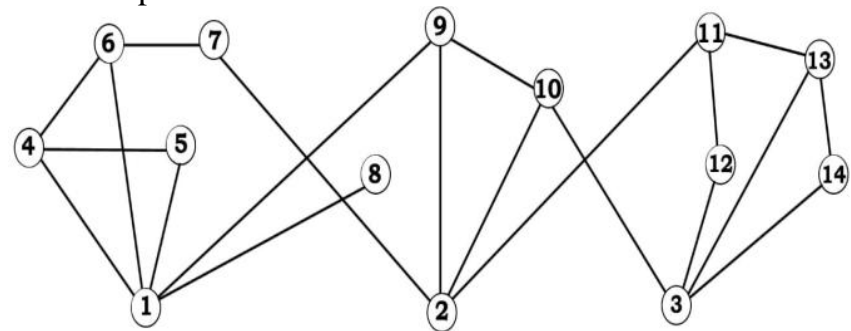

Fig : 13

Here dominating set $D=\{1,2,3\}$ and domination number is 3 . So it is enough to tag person 1 , person 2 and person 3 of university/college/office in order to make the post available to all the members.

\section{X.CONSEQUENCE OF THE PROBLEM OF RAMSEY}

If six people ( having Facebook account ) are randomly chosen then either three of the six people are friends with each other or three of the six people are not friends with each other. Take 6 randomly chosen people as vertices of a graph $G$, if two people are friends in $\mathrm{FB}$ then we draw a blue edge between their corresponding vertices and if they are not friends in FB then we draw a red edge between their corresponding vertices.

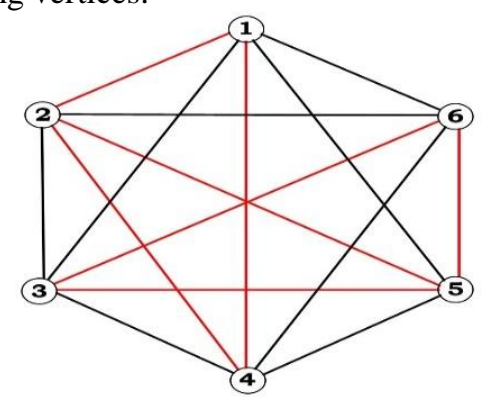

Fig : 14

Then we will see either a red triangle or a blue triangle in the graph $G$. Implying either three of them are friends with each other or three of them are not friends with each other.

\section{XI.COMPARISON BETWEEN GRAPHICAL STRUCTURE AND SOCIAL MEDIA NETWORK}

Consider any six person in a social media who are all friends with each other. Let $G$ be a graph where each of the six persons denotes vertices and

\section{Published By:}

Blue Eyes Intelligence Engineering $\&$ Sciences Publication

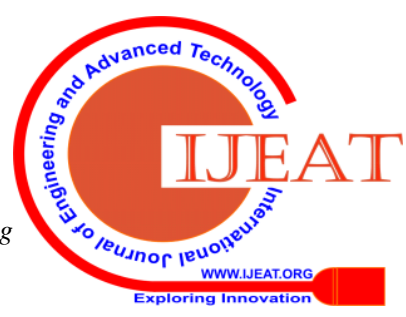


any two distinct vertices $x$ and $y$ are adjacent whenever there is a communication between the person $x$ and person $y$ in social media. Then by studying the graphical structure of social media network, we can easily made a conclusion about them.

1. If all the 6 persons are idle, that means there is no communication between any two of them then the corresponding graphical structure is a totally disconnected graph.

$(3)$

Fig : 15

For example : If all the 6 persons are students of same college/university and all of them are sitting in exam then we get this kind of graphical structure.

2. If any one of the 6 persons communicates with all but apart from that there is no communication between any two then the corresponding graphical structure is a star graph.

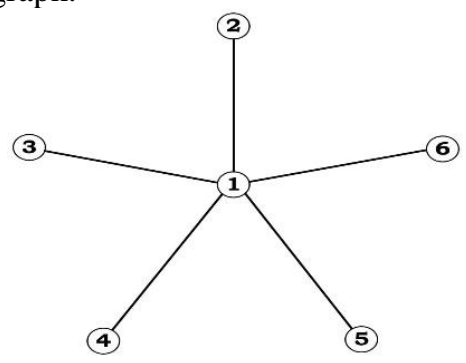

Fig : 16

For example : If person 1 invites all other persons in his/her birthday party by using social media then we get this kind of graphical structure.

3. If all the 6 persons communicates with each other then the corresponding graphical structure is a complete graph.

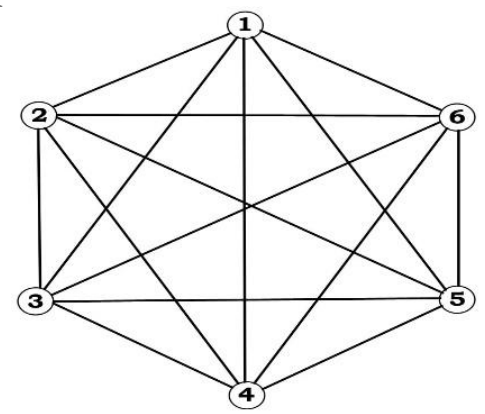

Fig : 17

For example : During new year or festivals, if everyone wishes each other through social media then we get this kind of graphical structure.

4. If person 1 communicates with person 2 , person 2 with person $3, \ldots \ldots$, person 6 with person 1 then the corresponding graphical structure is a cycle.

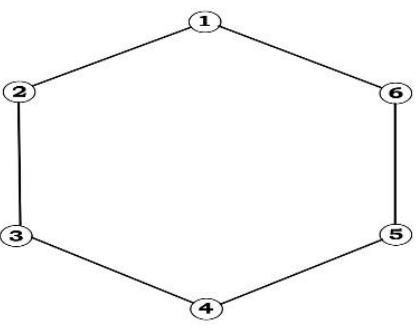

Fig : 18

For example : suppose person 1 buys two movie tickets and wants someone to watch the movie with him/her and so person 1 asked for it to person 2 , person 2 does not want to go so he/she asked person $3, \ldots \ldots$, finally person 6 asked person 1 without knowing that he/she is the one who has movie ticket then the corresponding graphical structure is like above.

\section{XII.RESULT AND DISCUSSION}

From the definition of measure of influence in a social graph we conclude that if a person $P_{1}$ is directly connected with more people as compared to person $p_{2}$ then that does not mean that the person $P_{1}$ is more influential than the person $p_{2}$. For example the CEO of a company is directly connected with few people yet he/she has the potential to influence more people than the others. Multi-connected social graph is preferred over single-connected social graph whenever there are two groups $G_{1}$ and $G_{2}$ and each group wants to have information from the other because in a single-connected social graph if the Duet-bridge between two groups is disrupted then there will be no way to have information whereas in multi-connected social graph we have other options. Among all the Duet-bridge present in a multi-connected social graph, we select that Duet-bridge which is connected to a person with higher measure of influence. The edge-cut helps us to minimize the study area of the graphical structure of the social media and with the help of the concepts of distance, diameter and girth of a social graph , we can determine the mutual acquaintances between two persons in a social media.The concepts of domination number and dominating set plays an important role to tackle the problem of tagging minimum number of persons in a post so that it will be available to every person in the network. For example: in Fig:13 if we tag person 1 , person 2 and person 3 in a post then that post is available to every persons present in the network. The consequence of the problem of Ramsey on a social graph is that whenever six persons are chosen from a social media then either three of them are friends with each other or three of them are not friends with each other.

\section{XIII.CONCLUSION}

The measure of influence of a particular person in a social network gives the potentiality of that person influencing more people in the network and thus helps in spreading or getting more information. A multi-connected social network graph gives more effective way to have information from different groups than a single connected social network graph. Triangular edge helps in determining the chances that the two unknown persons become friends with each other in future.

Published By:

Blue Eyes Intelligence Engineering

\& Sciences Publication

(C) Convriaht: All riahts reserved.

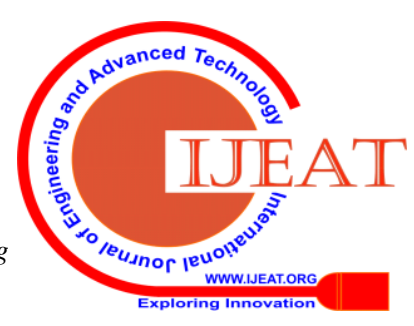


The domination number and dominating set play an important role in the social network graph since by tagging the minimum number of persons, a post can be made available to every persons in the network. The distance between two persons gives mutual acquaintances or mutual non-acquaintances between them. Similar results can also be obtain by determining girth and diameter of the social graph.

\section{REFERENCES}

1. Boyer, J.M.; Myrvold, W.J.: On the cutting edge : simplified o(n) planarity by edge addition. Journal of graph algorithm and applications, 2004, 8(3), 271-273

2. Carrington, P.J.; Scott, J.; Wasserman, S.: Models and methods in social network analysis. Cambridge; New York: Cambridge University press (2005).

3. Chakraborty, A.; Dutta , T.; Mondal, S.; Nath , A.: Application of graph theory in social media. International journal of computer sciences and Engineering, 2018, Vol-6, issue-10, E-ISSN : 2347-2693.

4. Harary, F.: Graph Theory, 1969 by Addison-Wesley publishing company, Inc.

5. Kala, R.; Kannan, A.: Social media netwoks and mathematics- a comparison. International journal of recent scientific research, 2017, 8(3), 15870-15872.

6. Kimball martin, "Graph theory and social networks spring 2014 notes" April 30 , 2014.

7. Mondal, B.; De, K.: A comparison of graph theory and social media network. International journal of current advanced research, 2018, 7(2(A)), 9649-9652.

8. Naidoo, J.; Kopung, K.J.: Exploring the use of WhatsApp in mathematics learning : A case study. Journal of communication, 2017, 266-273.

9. Sosial nettverksteori in blogtilhorende kurset INFO207/INF207 pa universitetet i Bergen , innlegg skrevet av studentene med

\section{AUTHORS PROFILE}

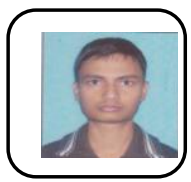

Kamal Jyoti Barman , PhD Research Scholar Department of Mathematics , Gauhati University , Guwahati-781014.

Educational Qualification : MSc (Gauhati University), PGDCA ( Gauhati University IDOL).Research Area : Graph Theory, Algebra .

E-mail : kamaljyotibarman@yahoo.in

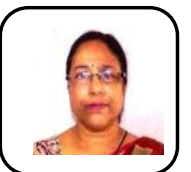

Kuntala Patra , Professor , Department of Mathematics , Gauhati University, Guwahati-781014.

Educational Qualification : MSc (Gauhati University), PhD (Gauhati University).

$$
\text { Research Area : Graph Theory , }
$$

Algebraic Topology , Algebra , Mathematical Education.

Teaching : Graph Theory, Algebra , Mathematical Logic , Operation

Research.E-mail : kuntalapatra@gmail.com

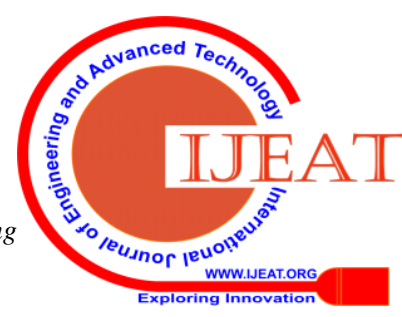

